\title{
Developmental Potential of Slowly Developing Embryo Like Morulae, Cavitating Morulae and their Further Management-A Short Communication
}

ISSN: 2640-9666

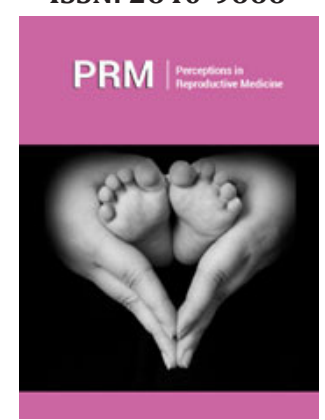

*Corresponding author: Kulvinder Kochar Kaur, India

Submission: 师 April 26, 2019

Published: 觜 May 15, 2019

Volume 3 - Issue 2

How to cite this article: Kulvinder Kochar K. Developmental Potential of Slowly Developing Embryo Like Morulae, Cavitating Morulae and their Further Management-A Short Communication. Perception in Reproductive Medicine.3(2). PRM.000558.2019.

DOI: 10.31031/PRM.2019.03.000558

Copyright@ Kulvinder Kochar K, This article is distributed under the terms of the Creative Commons Attribution 4.0 International License, which permits unrestricted use and redistribution provided that the original author and source are credited.

\section{Kulvinder Kochar $\mathbf{K}^{*}$}

India

\section{Short Communication}

The blastocyst stage is the last embryonic stage before implantation and is assumed to be the best stage of embryo selection compared to embryo at cleavage stage. Even when using the most advanced culture media not every embryo will reach the blastocyst stage and transfer them at day 5 [1]. From the time-lapse literature as regards to embryo selection that is successful outcome from the transfer of a blastocyst depends partially on it reaching that stage but even more that how and when it reached. Simple watching the emerging fluid filled space in a morula depends on the underlying works of a number of ordered molecular interactions. Blastulation is a complex process, with the resulting blastocyst representing the successful derivation of divergent cell lineages, a culmination of an ordered sequence of processes which begin with a "start signal", compaction, in mammalian embryos. Sozen B et al. [2] reviewed this process, explaining, how it begins as junctional complexes form at apico lateral and lateral sites on blastomeres, that is followed by polarization within the outer cell mass.

From these mouse studies what is clear is that cell polarity proteins (Par 1, Par3, APKC,

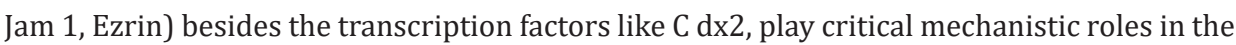
process. Mouse knockout studies show a complex genomic interplay which indicate that both maternal and zygotic $\mathrm{C} d x 2$ expression are needed for blastocyst formation from morulae [3]. In human embryogenesis also similar processes are present but blastocyst formation from the beginning of compaction is a progress at least in contemporary culture systems and one that either slows or even fails on a somewhat regular basis. Iwata $\mathrm{K}$ et al. [4] used timelapse cinematography to watch thawed human embryos, cryopreserved at early stages and observed that compaction was initiated at the eight-cell stage in $86.1 \%$ of embryos. Of these only roughly half developed into good quality blastocysts. If compaction began earlier as it did in $13.9 \%$ embryos the results were not even worse, with only $18.8 \%$ forming good quality blastocysts.

It is not easy to find why this occurs despite the tools for dynamic study of early embryogenesis like the time-lapse microscopy. Kirkegaard et al. [5] showed that changes in the temporal events of development is complicated probably caused by multiple factors instead of a single one with being dependent on patient factors heavily, hence origin of embryo is a big factor in the timing of preimplantation embryogenesis. They noted for example that the morpho kinetic parameters of time to initial blastocoel formation and time to full blastocoel formation are both affected by dose of FSH along with number of previous IVF attempts. Further patient age seemed to affect blastulation time to initial blastocoel formation occurred much later with older age. How, extrinsic factors modify the intrinsic factors controlling blastocyst formation remains unknown. Gradually with time our understanding of what are the requirements for the successful determination and differentiation of trophectoderm and inner cell lineages might allow us to intervene if they falter i.e., use of "precision embryology"? Currently we are faced with making decisions regarding what is to be done with slowly developing embryos sometimes they are just the ones available for transfer. 
Question arises should we discard do a fresh transfer culture further culture further and transfer, culture further and vitrify? This dilemma has been taken up by Hass et al. [6], who examined the developmental fate of delayed morulae the ones that form on day 5 vs those morulae that cavitate on day 5 . What is the importance of cavitation for further development. In this retrospective analysis of a large number of delayed embryos the authors showed that it depends and is not necessarily all that informative. Stopping on day 5 with a delayed embryo once another 24 to $48 \mathrm{hrs}$ of culture time is available for the embryo to continue to grow is not chosen in many ART programs. Yet Haas et al. [6] compared the pregnancy rates obtained from day 5 fresh transfers between pregnancy rates obtained from day 5 fresh transfer between delayed morulae and cavitating delayed morulae showed no statistical deference. But when each of these was cultured further until day 6 for potential vitrification, the delayed morulae produced significantly fewer blastocysts, only one in five and half the number compared with those cavitating on day 5 . These resulting blastocysts seemed to be of equivalent quality when vitrified and transferred at a future date, since the pregnancy rates were similar between the two groups.

Thus, the authors concluded that fresh transfer of delayed morulae is the alternative to continued culture and vitrification, that is a valuable information derived from a great number. Earlier it had been shown by Franasiak et al. [7] that either slowly developing embryos or asynchrony between embryo and endometrium leads to implantation failure. Further Tulat et al. [8] showed the importance of embryos carrying paternally inherited BRCA1 or 2 pathogenic variants were shown to develop more slowly compared with the maternally inherited BRCA1 or 2 pathogenic mutations. Thus, this study suggested that differential demethylation of parental genomes can influence the early development of preimplantation embryos. Expression of maternal and paternal genes is required for the completion of embryogenesis. The lesson to be gained from this study is as universally embryo culture gives subpar embryo all the time that is available regarding what we think is acceptable. Pregnancies getting achieved from the embryos cryopreserved on day 7 of culture attests that it is a reasonable approach. Supposedly no, in all the cases and timing of cavitation might indeed be one marker which projects embryonic fate upon continued culture vs stopping short with fresh transfer. Pool [9] compares this situation for embryologists professionally just like musicians, trapeze artists and demolition experts whereby decisions regarding timing are everything. Thus, embryos which gently apply the brakes might be very different from those that do so abruptly and thus our laboratory and clinical decisions should be informed by them instead of presuming that additional time used in culture gives additional quality. Yang et al. [10] showed that blastulation was not associated with increased aneuploidy rates, but the pregnancy rates with euploid day 5 embryos was statistically significant higher than with euploid day 6 blastocysts [10]. However Taylor TH [11] examined the euploidy rates and outcomes between day 5 and day 6 blastocysts and showed that day 5 and blastocysts had a higher chance of euploid than day 6 blastocysts, He also showed that when only euploid day 5 or euploid day 6 blastocysts were transferred during a cryopreserved embryo transfer , the cycle outcomes were similar.

\section{References}

1. Glujovsky D, Farquhar C, Quinteiro RAM, Alvarez SCR, Blake D (2016) Cleavage state versus blastocyst stage embryo transfer in assisted reproductive technology. Cochrane Database Sys Rev 6: CD002118.

2. Sozen B, Can A, Demir N (2014) Cell fate regulation during preimplantation development: A view of adhesion-linked molecular interactions. Dev Biol 395(1): 73-83.

3. Jedrusik A, Cox A, Wicher K, Glover D, Goetz ZM (2015) Maternal-zygotic knockout reveals a critical role of $\mathrm{Cdx} 2$ in the morula to blastocyst transition. Dev Biol 398(2): 147-152.

4. Iwata K, Yumoto K, Sugishim M, Mizoguchi C, Iba Y, et al. (2014) Analysis of compaction inhibition in human embryos by using time lapse cinematography. J Assist Reprod Genet 31(4): 421-426.

5. Kirkegaard K, Sundvall L, Erlandsen M, Hindkjaer JJ, Knudsn UB, et al. (2016) Timing of human preimplantation embryonic development is confounded by embryo origin. Hum Reprod 31(2): 324-331.

6. Haas J, Meriano J, Bassil R, Barzilay E, Zilberberg E, et al. (2019) Developmental potential of slow developing embryos: day 5 morulae compared with day 5 cavitating morulae. Fertil Steril 111(1): 105-111.

7. Franasiak JM, Alonso RM, Simon C (2016) Both slowly developing embryos and a variable pace of luteal endometrial progression may conspire to prevent normal birth in spite of a capable embryo. Fertil Steril 105(4): 861-864.

8. Tulay P, Doshi A, Serhal P, Gupta SSB (2016) Differential expression of parental alleles of BRCA1 in human preimplantation embryos. Eur J Hum Genet 25(1): 37-42.

9. Pool TB (2019) Commentary: When embryos hit the brakes. Fertil Steril 111: $48-49$.

10. Yang H, Yang Q, Dai S, Li G, Jin H, et al. (2016) Comparison of differences in development potentials between thawed D5 and D6 blastocysts and the relationship with pregnancy outcomes. J Assist Reprod Genet 33(7): 865-872.

11. Taylor TH, Patrick JL, Gitlin SA, Wilson JM, Cran JL, et al. (2014) Comparison of aneuploidy, pregnancy and live birth rates between day 5 and day 6 blastocysts. Reprod Biomed Online 29(3): 305-310. 